\title{
A Rare Case of Glossopharyngeal Neuralgia due to Neurovascular Conflict
}

\author{
Marc Vecchi $^{a} \quad$ Ricardo Pereira Mestre $^{b} \quad$ S. Luciamma Thiekalamuriyil ${ }^{c}$ \\ Roberto Cartolari ${ }^{d}$ \\ ${ }^{a}$ Centro Medico, Chiasso, Switzerland; ${ }^{b}$ Casa St. Lucia, Arzo, Switzerland; ${ }^{c}$ Department of \\ Internal Medicine, Ospedale San Giovanni - EOC, Bellinzona, Switzerland; ${ }^{d}$ Diagnostic and \\ Interventional Radiology Service, Ospedale San Giovanni - EOC, Bellinzona, Switzerland
}

\section{Keywords}

Neurovascular conflict $\cdot$ Neuralgia $\cdot$ Glossopharyngeal nerve $\cdot$ Facial pain · Pregabalin

\begin{abstract}
In the context of painful cranial neuropathies, a very rare cause is represented by the irritation of the glossopharyngeal nerve due to various aetiologic factors. Here, we present a case of neuralgia of the ninth right cranial nerve due to a compression of its nerve root upon the kinking of the homolateral vertebral artery, resulting in a disabling clinical overview for the patient. Our objective was to focus the reader's attention on the clinical manifestation, which alone could lead to an immediate diagnosis. The imaging and laboratory studies proved to be fundamental in diagnosing the causes, yet the knowledge of the symptoms and the signs of this rare clinical entity can prevent misdiagnosis, mismanagement and consequent economic expenditure as occurred in the case described here.

(C) 2017 The Author(s)

Published by S. Karger AG, Basel
\end{abstract}

\section{Introduction}

Facial pain is a highly disabling condition for the patient and often difficult for the clinician to interpret, with risk of misdiagnosis and mismanagement associated with severe 
stress for the patient involved and for the physician concerned. The causes are myriad, with very similar clinical presentations. There are also many possible anatomical structures that may be responsible and involved, such as bone, articular, vascular and nervous tissues.

\section{Case Report}

An 83-year-old man presented to our institution for the assessment of 3 months of intense and piercing pain, which was of an intermittent and paroxysmal nature, with a duration varying from a few seconds to a maximum of about $1 \mathrm{~min}$, of a daily frequency, localised in the deep laterocervical site behind the right jaw angle and the base of the homolateral tongue. The paroxysms were triggered and exacerbated by palpation localised at the root of the neck, by yawning, by tussive actions and by deglutition; they first occurred with solid food and then appeared with the swallowing of liquids, so as to have caused at anamnesis a weight loss of approximately 6-7 $\mathrm{kg}$ from the time the symptoms arose. These paroxysms tended to arise suddenly without prior notice and to regress spontaneously.

In the 3 months prior to our assessment, the patient had undergone various diagnostic tests and specialist consultations (native and with contrast agent cerebral CT scans, CT angiogram of the supra-aortic vessels, MRI of the cervical spine, 2 otorhinolaryngologist consultations with different specialists and a dental consultation which finally ended in dental extraction of the wisdom teeth without a clear diagnosis of the problem and without any therapeutic relief for the patient). The patient was submitted to our observation by the treating physician due to the appearance of the new symptom of hoarseness. The patient had a history of a hypertensive cardiopathy being treated with dihydropyridine calcium channel blockers, dyslipidaemia being treated with statin, diabetes being treated with Metformin and a pathological history characterised by a transient ischaemic attack in 1996 and a cervical spondylosis requiring treatment with paracetamol.

Objectively, we were faced with a disconsolate, sad and desperate patient; neuralgic pain was constant and severely exacerbated by deglutition of solids and liquids, by cough and local palpation. Incipient malnutrition was present. A careful neurological examination demonstrated a reduced sensitivity of the palatine veil, hoarseness and hyporeflexia of the pharyngeal reflex without distal neurological deficits. Vital signs were normal, he was normotensive, had a regular heart rhythm and normal ambient oxygen saturation. Laboratory findings were normal.

From a diagnostic point of view, considering the characteristics of the pain, its quality, the temporal characteristics and the location, and also substantiated by a precise neurological objective examination, we deduced a very high pre-test possibility of neuralgia associated with the ninth cranial (glossopharyngeal) nerve in. The diagnostic procedure was performed by MRI of the cranial base with fine cuts at the cerebral trunk level. This allowed for the determination of a compression by a vascular structure derived from the right vertebral artery named PICA (posterior inferior cerebellar artery) (Fig. 1, 2, 3).

The initial treatment according to specialist consultation was based on a conservative pharmacological approach by administering pregabalin, firstly at very low doses of 100 $\mathrm{mg} /$ day, followed by a therapeutic increase of $100 \mathrm{mg}$ every 3-4 days through to the maximum dose of $300 \mathrm{mg} /$ day.

The clinical progression was positive both in terms of tolerability of the therapy and in the antalgic therapeutic effect. The patient had progressively improved tolerability of parox- 
ysms, which became less frequent until their disappearance. Complete resolution of the symptomatology occurred in the third week of therapy and remained unchanged for 3 years.

\section{Discussion}

The clinical case described satisfies the clinical and imaging diagnostic criteria for neuralgia of the glossopharyngeal nerve [1], a condition characterized by repeated episodes of severe pain in the back of the tongue, in the back of the throat, part of the ear and tonsils. Attacks are brief and occur intermittently, from a few seconds to a few minutes, but they cause excruciating pain.

The ninth cranial nerve is constituted by parasympathetic secretory fibres directed at the parotid and by motor fibres directed at the stylopharyngeus muscle; by sensitive fibres directed at the back of the tongue and by sensitive fibres directed at the pharynx, tonsils, ear and tympanic membrane; finally, it consists of fibres that are attached to the carotid sinus and are, thus, involved in neurovegetative manifestations and syncopal attacks. Therefore, the main "triggers" that cause the typical "electric shock" pain are swallowing, coughing and yawning, which are often associated with neurovegetative manifestations, such as sialorrhea, pallor and syncopal attacks, which occur under a wide range of vasovagal terms but which are, in fact, secondary to a hyperstimulation of fibres of the ninth cranial nerve [2].

The diagnostic criteria for neuralgia of the glossopharyngeal nerve are represented by (1) the occurrence of at least 3 painful neuralgia attacks; (2) pain that should be present in the posterior region of the tongue, pharynx, mandibular angle and/or ear; (3) pain that is exacerbated by trigger points; and (4) in addition, no distal neurological deficits should be present [1]. The most frequent aetiologies are causes that may result in extrinsic compression, e.g., a tumour of the pontocerebellar angle, or neurovascular conflicts due to excessive tortuosity and ectasia of the vertebrobasilar area of a congenital nature or secondary to atherosclerotic phenomena of advanced age [3]. Such mechanical compression in line with the entry or exit zones of the nerve roots from the brain tract induce a functional hyperactivity of paroxysmal excitement of the nervous complex involved [2,4]. Secondly, inflammatory diseases (sarcoidosis, tuberculosis and Wegener's granulomatosis), infectious diseases, such as HIV, neurosyphilis and Borrelia, and demyelinating diseases (sclerosis plaques, syringobulbia and Wallenberg syndrome) must be named [4]. As far as our clinical case is concerned, in addition to MRI, we assessed the patient for serologies of the aforementioned virus, along with a serological haematochemical screening which also proved negative.

From an epidemiological point of view, the described neuralgia is very rare, representing a small percentage of patients, not only in the context of facial pain but also in the context of neuralgia of the cranial nerves [1]. In a retrospective epidemiological study [5], among all cases between 1945 and 1984, glossopharyngeal neuralgia had an incidence of 0.7 cases per 100,000 patients [5], while an epidemiological study conducted at the Mayo Clinic between 1922 and 1977 reported only 217 clinical cases in all these years [6].

Therapy is divided into conservative and surgical therapy. Generally, the first approach is of a conservative pharmacological nature using compounds capable of raising the threshold of nervous excitability. The pharmaceutical of choice is represented by carbamazepine, which is the most studied and evaluated drug with regard to neuropathic pain [7, 8]. Valid alternatives are represented by lamotrigine [9], gabapentin [10] and pregabalin [11]. In the event of ineffectiveness of single administration of carbamazepine, imbrication is advised with one of the aforementioned medications up to the therapeutically effective dosage [12]. 
Prolonged treatment is recommended for a period of at least 6 months, with regular checkups with a specialist in neurology, with progressive yet very slow reductions over time [12].

As far as surgical options are concerned, these should be taken into consideration either in cases when imbricated therapy of a conservative nature proves to be ineffective or when the aetiologic cause of neuralgia is such that it can be removed with neurosurgery, i.e., in percutaneous thermal ablation, chemical rhizolysis, ganglionic balloon compression, stereotactic radiosurgery and neurosurgical microdecompression $[12,13]$.

What should be underlined in the description of this clinical case is that the diagnosis of such a pathology is entirely clinical. The diagnosis both by serological, haematochemical and imaging tools must be supportive in order to determine the subsequent treatment. This is also the case for a wide range of facial pain [3], conditions which are not common in clinical practice but are likely to cause embarrassment in the treating medical staff if not adequately trained and equipped with information and experience [4].

In fact, these are clinical conditions that involve various disciplines, including neurology, neurosurgery, otorhinolaryngology, oncology, ophthalmology and internal medicine; this implies that diagnosis is often delayed and associated with significant limitations of the patient's quality of life [13]. It is of paramount importance to firstly identify the main features of the painful symptom, the type of onset and the pain quality, the temporal characteristics and possible precipitating factors (triggers). Symptoms can be classified into neurogenic, inflammatory and muscle and bone related [3].

We sum up that neurogenic pain has characteristics of "electric shock-like pain" that is of a stabbing quality, being acute and sudden, explosive, paroxysmal and of a precipitated temporal character and favoured by triggering factors [4]. Inflammatory pain, on the other hand, has a pressing quality, like a "weight," it tends to be consistent and unremitting over time, and it is generally aggravated by movements that increase local pressure (e.g., pounding). Finally, muscle and bone pain reminds of inflammatory characteristics but is often closely related to the movement of the muscular group involved [3].

In the first systematic review of epidemiological studies on neuropathic pain [14], considering neuralgias that affect the face, diabetic neuropathy was the most frequent diagnosis, followed by post-herpetic neuralgia and trigeminal neuralgia; glossopharyngeal neuralgia is only the last one to be contemplated.

The purpose of this study was to provide a tool for clinicians for a standardised approach to identifying the cause of painful neuropathic facial pain, attributing importance to the most common causes.

\section{Conclusions}

Glossopharyngeal neuralgia is a rare disease, the mere clinical presence of which must lead to a diagnosis (neuralgic pain below the tongue and mandibular angle, exacerbation triggers, etc.). Sometimes, it may present atypical symptoms (otodynia, syncope, pharyngodynia and hoarseness) due to a specific distribution branch being primarily affected.

The aetiopathogenesis often acknowledges ab extrinseco causes of compression (neurovascular conflict, expansive lesions). MRI turns out to be an indispensable neuroradiological test for the study of the cranial nerves, especially in the context of neurovascular conflict. There is both conservative and neurosurgical treatment.

From the point of view of a clinical diagnosis, the take home message we provide is to discriminate inflammatory pain from neuralgic pain; we consider this to be of paramount 
importance when dealing with facial pain. Concerning neurogenic pain, the most frequent causes to take into account in clinical practice are, therefore, (in order of relevance) diabetic neuropathy, post-herpetic neuropathy, trigeminal neuralgia and, finally, ninth cranial nerve neuropathy [15].

\section{Statement of Ethics}

The authors have no ethical conflicts to disclose.

\section{Disclosure Statement}

The authors have no conflicts of interest to declare.

\section{References}

1 Headache Classification Committee of the International Headache Society (IHS): The international classification of headache disorders, 3rd edition (beta version). Cephalalgia 2013;33:629-808.

2 Pellegrino M, Verlicchi A, Zanotti B, Alessandrini F, Nicolato A, Bricolo A: Glossopharyngeal neuralgia. Riv Neurorad 1999;12:567-574.

3 Schoenen J: Differential diagnosis of facial pain. Acta Neurol Belg 2001;101:6-9.

4 Siccoli MM, Bassetti CL, Sándor PS: Facial pain: clinical differential diagnosis. Lancet Neurol 2006;5:257-267.

5 Katusic S, Williams DB, Beard CM, Bergstralh EJ, Kurland LT: Epidemiology and clinical features of idiopathic trigeminal neuralgia and glossopharyngeal neuralgia: similarities and differences, Rochester, Minnesota, 1945-1984. Neuroepidemiology 1991;10:276-281.

6 Rushton JG, Stevens JC, Miller RH: Glossopharyngeal (vagoglossopharyngeal) neuralgia - a study of 217 cases. Arch Neurol 1981;38:201-205.

7 Aguggia M: Typical facial neuralgias. Neurol Sci 2005;26(suppl 2):s68-s70.

8 Reynes P, Soulet H, et al: Pain-dysfunction syndrome of the masticatory system curiously relieved by carbamazepine (Tegretol) (in French). Rev Odontostomatol (Paris) 1984;13:199-204.

-9 Titlic M, Jukic I, Tonkic A, Grani P, Jukic J: Use of lamotrigine in glossopharyngeal neuralgia: a case report. Headache 2006;46:167-169.

10 Moretti R, Torre P, Antonello R, Bava A, Cazzato G: Gabapentin treatment of glossopharyngeal neuralgia: a follow-up of four years of a single case. Eur J Pain 2002;6:403-407.

11 Kitchener JM: Glossopharyngeal neuralgia responding to pregabalin. Headache 2006;46:1307-1308.

12 Singh PM, Dehran M, Mohan VK, Trikha A, Kaur M: Analgesic efficacy and safety of medical therapy alone vs combined medical therapy and extra-oral glossopharyngeal nerve block in glossopharyngeal neuralgia. Pain Med 2013;14:93-102.

13 Zakrzewska JM: Facial pain: an update. Curr Opin Support Palliat Care 2009;3:125-130.

14 Van Hecke O, Austin SK, Khan RA, Smith BH, Torrance N: Neuropathic pain in the general population: a systematic review of epidemiological studies. Pain 2014;155:654-662.

15 Koopman JS, Dieleman JP, Huygen FJ, de Mos M, Martin CG, Sturkenboom MC: Incidence of facial pain in the general population. Pain 2009;147:122-127. 


\section{Case Reports in Neurology}

\begin{tabular}{l|l}
\hline Case Rep Neurol 2017:9:309-315 \\
\hline DOI: 10.1159/000485421 & $\begin{array}{l}\text { C } 2017 \text { The Author(s). Published by S. Karger AG, Basel } \\
\text { www.karger.com/crn }\end{array}$ \\
\hline Vecchi et al.: A Rare Case of Glossopharyngeal Neuralgia due to Neurovascular Conflict
\end{tabular}

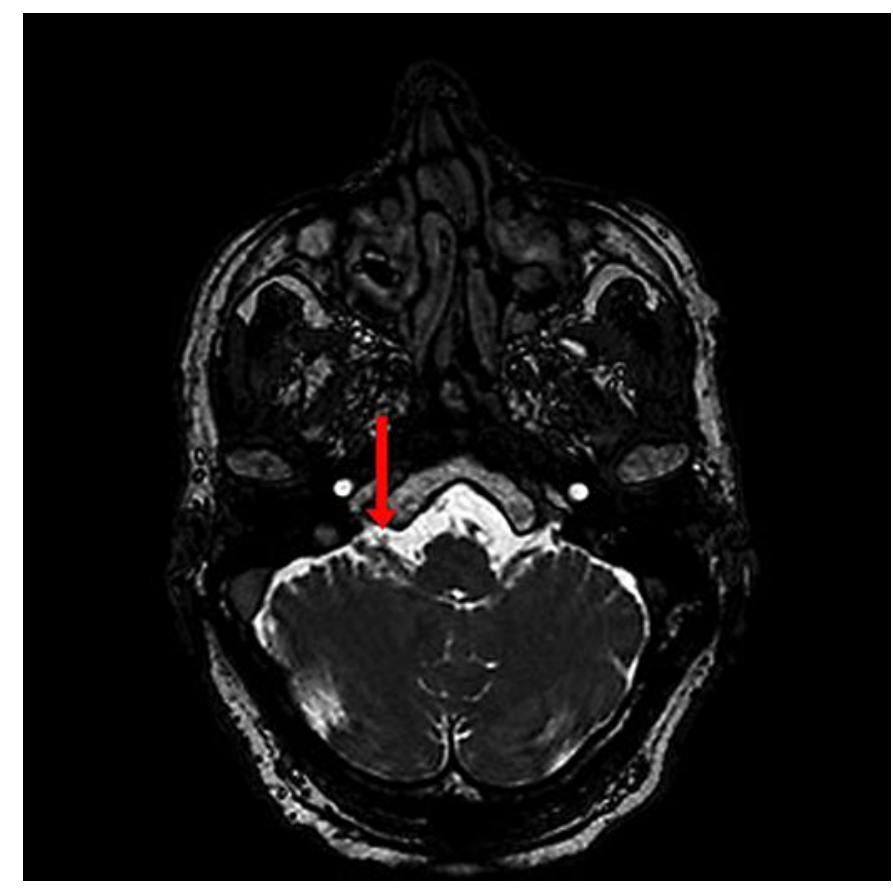

Fig. 1. The right glossopharyngeal nerve is compressed by a vascular structure (PICA).

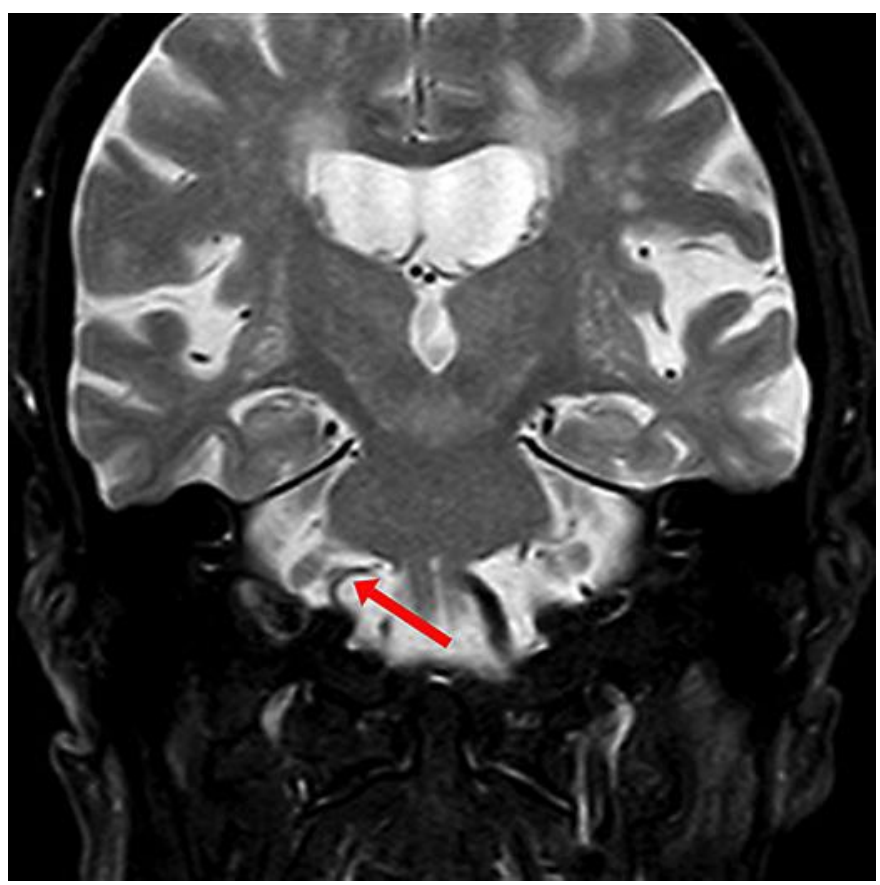

Fig. 2. Coronal T2W view: the PICA (arrow) compresses the right glossopharyngeal nerve. 


\section{Case Reports in Neurology}

\begin{tabular}{|c|c|}
\hline \multicolumn{2}{|c|}{ Case Rep Neurol 2017;9:309-315 } \\
\hline DOI: $10.1159 / 000485421$ & $\begin{array}{l}\text { ( ) } 2017 \text { The Author(s). Published by S. Karger AG, Basel } \\
\text { www.karger.com/crn }\end{array}$ \\
\hline
\end{tabular}

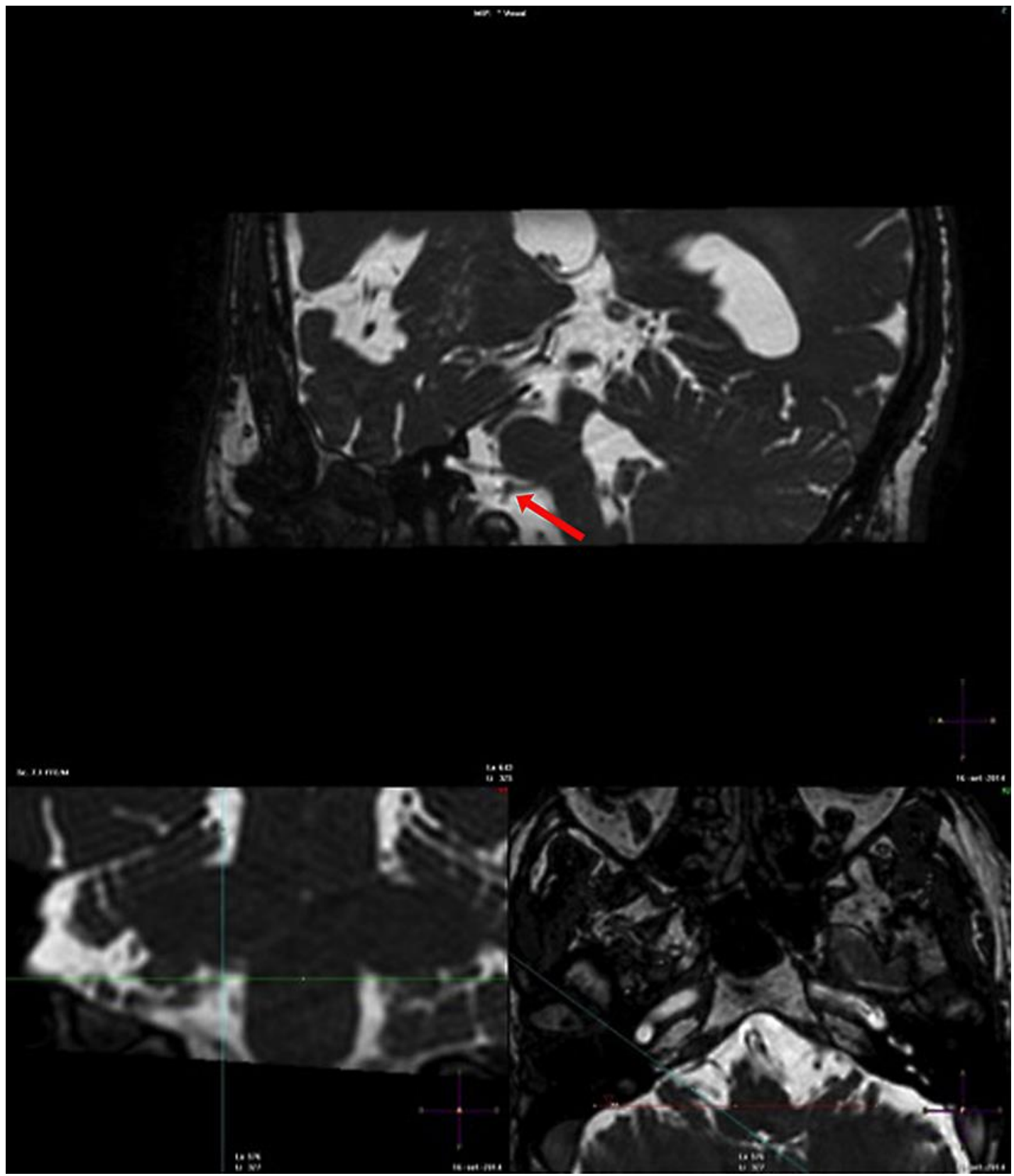

Fig. 3. T2W oblique para-sagittal view: the right glossopharyngeal nerve is crossed by PICA. 